\title{
ABSTRACTS OF PAPERS
}

\section{SUBMITTED FOR PRESENTATION TO THIS SOCIETY}

The following papers have been submitted to the Secretary and the Associate Secretaries of the Society for presentation at meetings of the Society. They are numbered serially throughout this volume. Cross-references to them in the reports of the meetings will give the number of this volume, the number of this issue, and the serial number of the abstract.

\section{Professor L. L. Dines and Dr. N. H. McCoy (National Research Fellow): On linear inequalities.}

In an address which appeared in this Bulletin (vol. 36, p. 393) one of the present authors discussed linear inequalities and some related properties of functions. Subsequently several contributions to this field have been made, and also important earlier contributions have become known to the present authors. The object of this paper is to discuss these various contributions with some attempt to correlate and amplify them, and to supply a more complete bibliography of the subject than has yet appeared. (Received August 8, 1931.)

279. Professor C. R. Adams: Transformations of double sequences, with application to Cesàro summability of double series.

Let $\left\|a_{m n k l}\right\|\left(m, n, k, l=1,2,3, \cdots ; a_{m n k l}=0\right.$ for $k>m$ or $l>n$ or both) be a matrix defining a transformation $A$ of double sequences. Necessary and sufficient conditions that $A$ be regular for the class of bounded sequences are known (Robison, Transactions of this Society, 1926); we show that if $A$ satisfies these conditions, it is regular for a much larger class of sequences. The case in which $a_{m n k l}=a_{m k}^{\prime} \cdot a_{n l}{ }^{\prime \prime}$, where $\left\|a_{m k}{ }^{\prime}\right\|$ and $\left\|a_{n l}{ }^{\prime}\right\|$ are triangular matrices defining transformations $A^{\prime}$ and $A^{\prime \prime}$ of simple sequences, is of special interest from the standpoint of summability of double series. We then write $A=A^{\prime} \cdot A^{\prime \prime}$ and prove that if $A^{\prime}$ and $A^{\prime \prime}$ are both regular, $A$ is regular for the class of double sequences of which each row is transformed by $A^{\prime \prime}$, and each column by $A^{\prime}$, into a bounded sequence. From this flows a consistency theorem for Cesàro summability of double series which is of greater generality than those previously given. Extensions of the main result to the case in which $A^{\prime}$ and $A^{\prime \prime}$ are defined by square matrices, to the case in which $A^{\prime}$ and $A^{\prime \prime}$ are merely convergence-preserving, to general consistency questions as discussed by Hurwitz and Silverman (Transactions of this Society, 1917), and to sequences of multiplicity $n>2$, are immediate. (Received August 4, 1931.)

280. Professor G.C. Evans: The Cauchy integral and summable boundary functions. 
The author obtains necessary and sufficient conditions that the function $w(z)=(2 \pi i)^{-1} \int[f(t)+i g(t)](t-z)^{-1} d t$, in the region $T$ bounded by $C$, take on the boundary values $f(t)+i g(t)$ for approach in the wide sense to the boundary $C$, at a point set of positive measure. The functions $f(t), g(t)$ are real and summable $(L)$ on $C$, where $C$ is a simple closed curve, to which the Fredholm analysis of the Dirichlet problem is applicable. A necessary and sufficient condition is that $w(z)$ vanish identically outside $C$. An equivalent condition is that $w(z)$ be representable in terms of a potential of a double layer for a mass distribution which is absolutely continuous on $C$, or that the absolute continuity of $\int|w(z)||d z|$ over curves $C^{\prime}$ neighboring $C$ and satisfying conditions analogous to those specified by Evans and Miles (American Journal of Mathematics, vol. 53, (1931), pp.493-516), be uniform as $C^{\prime}$ approaches $C$. The class of these functions are equivalent to the class represented by a Cauchy integral with the alternative boundary conditions already described by the author (Cleveland Meeting, Dec. 1930). A representation in terms of the Green's function and its conjugate is permissible. Among such curves $C$ the representation is invariant under a conformal transformation. (Received August 10,1931.)

281. Professors C. L. E. Moore and Philip Franklin: Dual Pfaffians.

A Pfaffian expresses the vanishing of a linear combination of differentials which are the components of a vector. When these are replaced by the components of an $(n-1)$-dimensional element, we have a dual configuration. Similarly we have dual expressions in $m$ - and $(n-m)$-dimensional elements. In this paper we develop the geometry of the configurations connected with such expressions. (Received July 28, 1931.)

\section{Professor C. V. Newsom: On the behavior of entire func- tions in distant portions of the plane.}

This paper represents an extension and a generalization of the investigations of W. B. Ford and E. W. Barnes on the asymptotic representation of entire functions when such a function is regarded as given through its Maclaurin's development. Under rather liberal restrictions upon the general coefficient of the given series it is found that the function thus defined is asymptotic to a certain definite integral. The method of the investigation, as in the work of Ford and Barnes, involves the Cauchy integral theorem and contour integration. (Received August 5, 1931.)

\section{Professor C. N. Moore: On sets of constants which are not the Fourier constants of a function integrable $(L)$.}

The purpose of this paper is to set up a general method of constructing a set of constants, $a_{n},(n=0,1,2, \cdots)$, where $\lim _{n \rightarrow \infty} a_{n}=0$, but such that the trigonometric series $\sum a_{n} \cos n x$ is not the Fourier development of a function that is integrable $(L)$. In many cases the trigonometric series can be shown to converge throughout any interval of length $2 \pi$, except at a finite number of points. In the neighborhood of one or more of these points, however, the func- 
tion represented by the series becomes infinite to a sufficiently high degree to cause its integral to diverge. Simple cases of such series are the following:

$$
\begin{gathered}
(\cos 3 x) / \log 3+(\cos 7 x) / \log 7+(\cos 11 x) / \log 11+\cdots, \\
(\cos 7 x) / \log 7+(\cos 13 x) / \log 13+(\cos 20 x) / \log 20+\cdots .
\end{gathered}
$$

These examples are of interest because the series $\sum_{n=2}^{\infty}(\cos n x) / \log n$ is known to represent a function which has a Lebesgue integral over any finite interval. (Received August 10, 1931.)

\section{Professor R. E. Langer: On the asymptotic forms of the solutions of differential equations.}

In a recent paper the author has discussed the asymptotic forms of the solutions of the differential equation (1) $d^{2} u / d x^{2}+\lambda^{2} \phi(x) u(x)=0$, under the hypotheses: (i) that $x$ is real, $a \leqq x \leqq b$; and (ii) that $\phi(x)=\left(x-x_{0}\right)^{\nu} \phi_{1}(x)$, in which $\phi_{1}(x)$ is real and different from zero on $(a, b)$, while $a<x_{0}<b$, and $\nu \geqq 0$. This investigation is extended in the present paper to a discussion of the forms of the solutions when the variable $x$ is complex and varies over a region of the complex plane which includes the point $x_{0}$. The restriction that $\phi_{1}(x)$ be real (that is, of constant argument) is also removed. Inasmuch as the Bessel equation is expressible in the form (1), the results obtained may, in particular, be specialized to yield the asymptotic forms of the Bessel functions $J_{ \pm \rho}(z)$ for all complex values of the parameter $\rho$ and the argument z. (Received August 5, 1931.)

285. Professor R. E. Langer and Dr. M. L. Hartung: On a class of integral equations with a finite discontinuity in the kernel.

This paper deals with the integral equation $u(x)=\lambda \int_{-\alpha}^{\alpha} K(x, \xi) u(\xi) d \xi$ whose kernel $K(x, \xi)$ has a finite non-vanishing discontinuity along the line $\xi=-x$. In a sequence of papers published individually and jointly in the Transactions of this Society between 1926 and 1928, Langer and Tamarkin have developed a theory of this equation based upon a discontinuity in the kernel or its derivatives when $\xi=x$. The extent to which the specialized location of this discontinuity governs the development of the theory has hitherto remained open. It is proved in this paper under hypotheses of a more or less general nature that there exist infinitely many characteristic values of the parameter $\lambda$, and asymptotic expressions for these values and for the characteristic functions are obtained. The problem of the expansion of an arbitrary function in a series of the solutions is also discussed. The method used consists of a transformation of the given equation into a functional system which is studied by an adaptation of methods from the theory of differential equations. Methods and results are analogous to those of the papers mentioned above and might tend to show that the location of the discontinuity may not be of major importance. (Re. ceived July 31,1931 .)

286. Professor Norman Miller: Some extensions of the Schwarz. Stieltjes theorem of mean value and some consequences deducec therefrom. 
Among several generalizations of the mean value theorem of the differential calculus one was given by Schwarz and proved in a simple manner by Stieltjes. Using Stieltjes' method of proof some further generalizations are here stated together with particular cases of which Lagrange's interpolation formula is one. The main results of the paper flow from these theorems by limiting processes. These culminate in a generalization of Taylor's theorem with the remainder involving an arbitrary number of functions and an arbitrary number of points. (Received July 31, 1931.)

287. Professor L. M. Graves: On the condition of Weierstrass in the problem of Lagrange.

It is usual in the calculus of variations first to derive the differential equations which a minimizing arc must satisfy, and then to derive the Weierstrass condition by a separate construction. In the case of the isoperimetric or the Lagrange problem, this construction requires the assumption that sub-arcs of the minimizing arc are normal. The author gives a new method of proof which depends on introducing strong and weak variations simultaneously. The new proof is shorter, and it is necessary to assume only that the minimizing arc as a whole is normal. The method is still applicable when the side conditions involve integral equations. For the isoperimetric problem the method yields also a new condition in the abnormal case, provided the arc is an extremal for the integral to be minimized as well as for the integral to be kept constant. (Received July 27,1931 .)

288. Dr. W. T. Reid: A boundary value problem associated with the calculus of variations.

In this paper is treated the boundary value problem that arises when one seeks to minimize $I_{2}(\eta)=2 H\left(\eta_{1}\left(x_{1}\right), \cdots, \quad \eta_{n}\left(x_{1}\right), \quad \eta_{1}\left(x_{2}\right), \cdots, \eta_{n}\left(x_{2}\right)\right)+$ $\int_{x_{1}}^{x_{2}} 2 \omega\left(x, \eta_{1}, \cdots, \eta_{n}, \eta_{1}^{\prime}, \cdots, \eta_{n}^{\prime}\right) d x$, where $H$ and $\omega$ are quadratic in their arguments, in the class of arcs $\eta$ which satisfy certain linear differential equations of the first order and certain linear end conditions, and which give a fixed value to the integral $\int_{x_{1}}^{x_{2}} \eta_{i}(x) K_{i j}(x) \eta_{j}(x) d x$. By considering the minimum of $I_{2}(\eta)$ in successive classes of admissible arcs the existence of infinitely many characteristic numbers for the boundary value problem is established when the matrix $\left\|K_{i j}\right\|$ satisfies rather general conditions. There are also given some expansion theorems in terms of the characteristic solutions. A boundary value problem of the type considered in this paper, and in which $\left\|K_{i j}\right\|$ reduces to the unit matrix, has recently been treated by Morse in the study of sufficient conditions for the problems of Lagrange and Mayer with variable end points; for such problems the second variation has the form $I_{2}(\eta)$ when the non-tangency condition is satisfied (M. Morse, American Journal of Mathematics, vol. 53 (1931), pp. 517-546). (Received August 7, 1931.)

289. Professor C. R. Adams: Note on multiple Dirichlet and multiple factorial series.

A recent paper (Annals of Mathematics, 1931,pp. 67-82) in which the writer developed some of the fundamental properties of multiple factorial series left 
open the question of the validity for multiple series of the natural analogue of the well known theorem concerning simple series: A factorial series in $x$ and the associated Dirichlet series both converge or both diverge at each point $x(\neq 0,-1,-2, \cdots)$ of the complex plane. It turns out that the natural analog is not valid, and the purpose of this note is to investigate completely the relation between the regions of convergence of the two associated multiple series. It is first shown that the region of convergence of the double Dirichlet series $\sum_{i, j=1}^{\infty} c_{i} e^{-\lambda_{i} x-\mu_{j} y}$, like that of the double factorial series, is a pair of related right half-planes. The interiors of the regions of convergence of the two associated series are then identified, after which it is proved by example that one series may converge and the other diverge at a boundary point. The extension to series of multiplicity $n>2$ is immediate. (Received August 4,1931.)

\section{Professor J. A. Shohat: On Stieltjes continued fractions.}

Let $\psi(x)$ be monotonic and non-decreasing on $(a, b)(b \geqq a>0, b$ finite or infinite). Let us consider the Stieltjes continued fraction for $\int_{a}^{b} d y(y) /(x-y)$ $=1 /\left(e_{1} x+1 /\left(e_{2}+1 /\left(e_{3} x \cdots\right) \cdots\right) \cdots\right),\left(e_{i}=\right.$ const. $)$. The object of the present paper is to investigate the relation of the denominators of its odd convergents to those of its even convergents, the latter being well known as the orthogonal Tchebycheff polynomials $\phi_{n}(x)$ for which $\int_{a}^{b} \phi_{m}(x) p_{n}(x) d x(x)=0,(m \neq n)$. The relation in question is found to be very simple and leads to some interesting applications in mechanical quadratures. The paper will appear in full in the American Journal of Mathematics. (Received July 15, 1931.)

\section{Professor J. A. Shohat: On some applications of Parse-} val's formula.

Let $\left\{f_{n}(x)\right\},(n=0,1,2, \cdots)$, be a system of orthogonal normal functions on $(a, b)$, for which Parseval's formula holds true: $\int_{a}^{b} f^{2}(x) d x=\sum_{n=0}^{\infty}\left(\int_{a}^{b} f(x) f_{n}(x) d x\right)^{2}$. By specifying $f(x)$ some interesting results are obtained. In particular, taking for $\left\{f_{n}(x)\right\}$ the polynomials of Legendre, Laguerre, etc. yields the sum of certain numerical series, as well as the solution of certain types of problems in the calculus of variations. (Received July 15, 1931.)

\section{Professor Harry Bateman: The k-function, a particular case of the confluent hypergeometric function.}

The $k$-functions satisfy the differential equation $x y^{\prime \prime}=(x-n)$ y and form a special class of confluent hypergeometric functions mentioned by Whittaker as needing investigation. By using a definite integral to define the $k$-function, its properties are easily developed and some definite integrals evaluated. An interpolation formula provides an alternative method of development. (Received June $13,1931$.

\section{Professor J. D. Tamarkin: On a theorem of S. Bernstein-} Widder.

An important theorem giving necessary and sufficient conditions for the representability of a function by means of a Laplace-Stieltjes integral was discovered recently by $S$. Bernstein and, subsequently but independently, by $D$. 
V. Widder. Widder's proof is essentially simpler than, and is based on considerations entirely different from Bernstein's. In the present note, which was suggested by the reading of Widder's paper which will appear in the Transactions of this Society, it is shown that Widder's proof can be made still simpler and shorter. (Received August 5, 1931.)

\section{Professor G. C. Evans: Complexes and dimension theory.}

The author constructs two systems of postulates for a theory of dimension, in each case independent among themselves. The dimension is defined for every set, but is not always the same as that of Menger, although the dimension of a continuous arc is conserved as unity. The postulates are, of course, based on those of Menger, but isotopic transformations are used instead of homeomorphic, and complexes instead of compact sets. In one system a denumerable set has positive dimension and the set of all rational points in $R_{3}$ has dimension 3 ; in the other system, dimension zero. In neither system is a set of positive measure $(L)$ in $R_{3}$ necessarily of dimension 3. (Received August 10,1931.)

295. Professor W. E. Roth: On the equation $P(A, X)=0$ in matrices.

The equation

$$
P(A, X) \equiv \sum_{k=0}^{D} F_{k}(A) X^{p-k}=0,
$$

where $F_{k}(\lambda), k=0,1, \cdots, p$, are polynomials or analytic functions in $\lambda$ and where $A$ and $X$ are square matrices, is here solved for $X$. Inequalities limiting the number and degree of the elementary divisors of the characteristic determinant, $|X-\mu I|$, of a solution are established. They depend only upon the character of the polynomial $P(\lambda, \mu)$ and upon the number and degree of the elementary divisors of $|A-\lambda I|$. Solutions of the given equation are considered under the hypotheses: namely, (a) that $X$ be a unilateral solution, (b) that $X$ be a bilateral solution, and (c) that $X$ be commutative with $A$. The effect of these successive restrictions upon $X$ is clarified and the difficulties which must be overcome to establish necessary and sufficient conditions for the existence of solutions under any of these hypotheses are exposed. (Received August 5, 1931.)

296. Professor C. G. Latimer: A correspondence between classes of matrices and classes of ideals.

All matrices considered in this paper are square matrices of the same order $n$. Unless otherwise stated, the matrices have rational integral elements only. The matrices $A, A_{1}$ will be said to belong to the same class if there is a matrix $Z,|Z|= \pm 1$, such that $A_{1}=Z A Z^{-1}$. Let $\phi(x)=x^{n}+c_{1} x^{n-1}+\cdots+c_{n}$ be irreducible, the $c$ 's being rational integers. If $\phi(A)=0$, then $\phi\left(A_{1}\right)=0$. We show that there is a one-to-one correspondence between the classes of matric solutions of $\phi(x)=0$ and the classes of ideals in the algebraic field defined by a root of the same equation. This result is extended to the case where the $c$ 's are integers in an algebraic field $f$, of class number unity. This paper will appear in vol. 33 (1932) of the Annals of Mathematics. (Received August 10,1931.) 
297. Professor H. S. Vandiver: On the method of infinite descent and Fermat's last theorem for regular prime exponents.

In this paper the author applies the method of infinite descent to obtain a proof of the Last Theorem for regular prime exponents in the first case of the theorem, that is, when in the relation $x^{\chi}+y^{\chi}+z^{\chi}=0, x y z$ is prime to the odd prime $\chi$. Heretofore the method of infinite descent has been used only for the proof of the theorem when one of the integers $x, y, z$ is divisible by $x$. (Received August 6,1931.)

\section{Professor W. L. Ayres: Note on a property of continuous} arcs.

In a recent paper (Proceedings Cambridge Philosophical Society, vol. 26, p. 31) Mr. S. Verblunsky proves the following result. Let $C$ be a continuous arc in the plane joining the points $p$ and $q$ and suppose that $C$ does not cross the line $p q$. Then given any positive number $h \leqq \rho(p, q)$ there exist two points $b$ and $c$ of $C$ such that $\rho(b, c)=h$ and the line $b c$ is parallel to the line $p q$. His proof involves properties of the function $\log (z-a)$ in the complex plane and the fact that an arc may be approximated by a sequence of polygonal lines of a certain type. In this note a simple proof is given following directly from the order properties of points on an arc. Also the theorem is generalized by replacing the arc by any bounded continuum and replacing the condition that $C$ does not cross the line $p q$ by the hypothesis that no point of the segment $(p, q)$ is a limit point of points of $C$ lying on one side of the line $p q$. (Received July 24, 1931.)

299. Dr. E. W. Miller: On a kind of homogeneity in continuous curves.

A continuum $C$ will be called homogeneous with respect to points of the same (Urysohn-Menger) order if for every two points $p$ and $q$ of $C$ which are of the same order there is a $(1-1)$ bicontinuous transformation $T$ of $C$ into itself such that $T(p)=q$. In this paper conditions are given which are necessary and sufficient that an acyclic continuous curve have the above property. (Received August 7, 1931.)

300. Mr. L. E. Bush: Algebras without moduli which are equivalent to the algebras of their first and their second matrices.

The purpose of this investigation is to answer the question as to the existence of linear associative algebras over an infinite field which, although not possessing moduli, are equivalent to the algebras of both their first and their second matrices. The index of an element of an algebra with respect to the algebra is defined and some of its properties are found. By the use of these indices and the decomposition of the algebra with respect to a principal idempotent element certain necessary conditions are derived for an algebra to be of the kind sought. With the aid of these an example of such an algebra is constructed. For a certain special type of algebras both necessary and sufficient conditions are found. (Received July 26, 1931.) 
301. Professor H. Hasse: Theory of cyclic algebras over an algebraic field.

Cyclic (Dickson) algebras over an algebraic field $\Omega$ of finite degree and defined by a cyclic field $Z$ of order $n$ and a number $\alpha$ in $\Omega$ are considered. The problem of characterizing such an algebra by means of a system of total invariants is completely solved. These results are applied to prove that the exponent of any cyclic algebra is its index, that the direct product of any two cyclic algebras is a cyclic algebra, and that the Dickson-Wedderburn sufficient condition that a cyclic algebra be a division algebra is also necessary. Finally it is shown that if an algebra is cyclically representable it is also cyclic. (Received August 3, 1931.)

\section{Professor Raymond Garver: On rational transformations.}

When a rational (non-integral) transformation is applied to the roots of an algebraic equation the setting up of the transformed equation is usually a rather cumbersome matter. This paper discusses devices which are useful when the denominator of the transformation is of a certain type. An important transformation on the Brioschi quintic is given special study. (Received August 10, 1931.)

303. Professor H. S. Vandiver: On the second factor of the class number of a cyclotomic field.

In this paper the author proves the following theorem. A necessary and sufficient condition that the second factor of the class number of the field defined by $\zeta=e^{2 i \pi / l}$ be divisible by $l$ is that at least one of the units $E_{i},\left(i=1,2, \cdots, l_{1}\right.$; $\left.l_{1}=(l-3) / 2\right)$, be the $l$ th power of a unit in $k(\zeta)$. Here $E_{i}=\epsilon^{R} ; R=r^{l^{2}}+s r^{l^{2}-2 i}$ $+s^{2} r^{2}{ }^{2}-4 i+\cdots+s^{l_{1}} r^{2}-2 i l_{1} ; \quad \epsilon=\left\{\left(1-\zeta^{r}\right)\left(1-\zeta^{-r}\right) /\left((1-\zeta)\left(1-\zeta^{-1}\right)\right)\right\}^{1 / 2} ; \quad r$ is a primitive root of $l$ and we are employing the Kronecker-Hilbert notation of symbolic powers, the substitution $s$ denoting $\left(\zeta / \zeta^{r}\right)$. An abstract of this paper appeared in the Proceedings of the National Academy of Sciences (vol. 16 (1930), pp. 743-749). (Received August 6, 1931.)

304. Professor H. S. Vandiver: On the norm-residue symbol in the theory of cyclotomic fields.

The norm-residue symbol (Hilbert, Sitzungsberichte, D. M. V., 1894, p. 413) has been treated by a number of writers, but so far, apparently, its value has always been represented by a summation. In this note the author gives a method for carrying out these summations. An abstract of this article appeared in the Proceedings of the National Academy of Sciences, vol. 16 (1930), pp. 740-743. (Received August 6, 1931.)

305. Professor H. S. Vandiver: Summary of results and proofs on Fermat's Last Theorem. (Sixth paper.)

In this article the author states a number of new results concerning the Last Theorem with proofs indicated in most cases. Theorems in articles already published are shown to yield proofs of the Last Theorem for all exponents less than 307. (Received August 6, 1931.) 
306. Mr. C. H. Harry: Concerning non-locally compact spaces.

In his paper The cyclic and higher connectivity of locally connected spaces (American Journal of Mathematics, vol. 53, No. 2, April, 1931, p. 442, Prob. (10.2)) G. T. Whyburn raises the question as to whether or not, given any two points $a$ and $b$ of a locally connected and connected space $M$ having no cut point, two mutually exclusive connected open sets $R_{1}$ and $R_{2}$ exist such that $a+b \subset \bar{R}_{1} \cdot \bar{R}_{2}$. The present paper contains an example showing that this is not always possible. Also included in the paper is the following theorem: If $M$ is any connected and locally connected space having no cut point and $a$ any point of $M$ such that $a$ together with no other point $z$ separates the space, then for any two other points $x$ and $y$ neither of which is a local end point there exists a region $R$, containing $a$, such that $x$ and $y$ lie together in the same component $C$ of $M-\bar{R}$, and furthermore, no point $p$ separates $x$ and $y$ in $C$. (Received August 5, 1931.)

307. Professor W. A. Wilson: On cyclic numbers of onedimensional compact sets.

In this paper is given a modification of Alexandroff's definition of the connectivity number of a one-dimensional compact continuum. This is used to derive theorems relative to the cyclic numbers of a one-dimensional compact set and its components and the divisor of a sequence of such sets, and an addition theorem valid for sets whose cyclic and component numbers are finite. (Received July 13, 1931.)

\section{Professor W. A. Wilson: On upper semi-continuous de- compositions of compact continua.}

It is shown that for a certain class of compact continua there is a unique upper semi-continuous decomposition into certain disjoint sub-continua as elements such that the original continuum is locally connected about each element and the decomposition is the finest possible such decomposition. The elements are sub-continua composed of oscillatory sets of the given continuum and the class of continua for which the theorems are valid embraces all simply connected one-dimensional compact continua as a sub-class. (Received July 13, 1931.)

309. Professor G. T. Whyburn: A junction property of locally connected sets.

In this paper it is shown that if $M$ is any connected locally connected separable metric set and $K$ is any self-compact subset of $M$, then $M$ contains a subset $H$ which is dense in $K$ and is the image under uniformly continuous transformation of the set $D$ of all dyadic rational numbers on an interval. As a corollary we have that if $M$ is a subset of some complete space, then $\bar{H}$ is a compact locally connected continuum (since it is the continuous image of an interval) and of course it contains $K$. With the aid of our theorem we deduce the Moore-Menger theorem on the arcwise connectivity of complete locally connected sets from the well known arcwise connectivity of compact locally 
connected continua. Also we prove in this paper that if $A$ and $B$ are relatively closed sets such that $A+B$ has $k$ components and $A \cdot B$ has $n$ components then $A$ and $B$ themselves have finite numbers $n_{a}$ and $n_{b}$ respectively of components and $n_{a}+n_{b} \leqq n+k$. (Received August 10, 1931.)

310. Professor G. T. Whyburn: Regular chains and their application to proofs in point set theory.

For any $\epsilon>0$, a connected set $C$ which is the sum of a finite number of $\epsilon$-continua no two having infinitely many points in common will be called a regular $\epsilon$-chain. The existence of locally connected regular $\epsilon$-chains joining any two given points in very general locally connected sets is readily established. Also it follows at once that the product of any monotone decreasing sequence of regular $\epsilon$-chains is regular if $\epsilon \rightarrow 0$ and thus, when connected, is locally connected. Advantage is taken of these facts to obtain very simple and easily followed proofs for the arcwise connectivity of locally connected continua and for the more general Moore-Menger theorem in complete spaces. (Received August 10, 1931.)

\section{Professor A. J. Maria: Examples of harmonic functions.}

The author adapts an entire function of Malmquist to show the existence of a not identically vanishing function, harmonic in the unit circle, which approaches, in the wide sense, zero at each boundary point. An example is also given for the sphere. It is further shown, by means of an example, that a function, analytic in the unit circle, which takes on, in the wide sense, summable boundary values at each point, is not necessarily given by a Cauchy integral. (Received August 10, 1931.)

\section{Dr. R. S. Burington: An invariantive classification of plane cubic curves under the affine group.}

The first classification of cubic curves given by Newton is open to objection on a number of grounds: (1) degenerate cases are not treated; (2) the classification is not invariantive and harbors certain ambiguities; (3) the rules for distinguishing species are unsatisfactory. In this paper we use as a basis for an invariantive classification of plane cubic curves under affine transformations a certain conic covariant $C_{2}$. (See Annals of Mathematics, December, 1928, pp. 52-60.) The cubic is reduced by means of the affine group to 42 canonical forms in 8 cases which are further divided into 110 species. Case $I$ is divided according to the types of diameters which the cubic has for chords parallel to each asymptote $A_{i}$. Each $A_{i}$ leads to a certain quartic covariant $E_{\boldsymbol{i}}(x)=0$ and associated discriminant matrix $M_{i}$ whose rank and signature are used extensively to separate cubics into species. The other cases are treated similarly. An extended abstract of this paper will appear in "Abstracts of Dissertations" (Ph.D), The Ohio State University Press, June 1931, No. 6. (Received July 29, 1931.)

313. Professor H. V. Craig: On a covariant differentiation process. 
This paper deals with a covariant differentiation process for functions which involve, in addition to the coordinate variables, derivatives of these variables with respect to a parameter. The process is based on a connection due to J. H. Taylor. (Received July 29, 1931.)

\section{Professor Lincoln LaPaz: Characteristic properties of the} euclidean length integral.

In a paper which will soon appear in the Proceedings of the National Acaddemy of Sciences, the writer has proved that the problem of minimizing the euclidean length integral is the only one for which transversality is orthogonality and the extremals are straight lines. In the present paper an independent proof of this result is given by a method of importance in the study of those inverse problems of the calculus of variations in which the extremal system is prescribed before any specification is made of the transversality relation as, for example, in the case of the Hamel geometries. (Received August 7, 1931.)

\section{Professor C. J. Coe: Vector treatment of the displacements of a rigid body.}

This paper presents what are believed to be new and very simple vector proofs of the basic theorems concerning the finite displacements of a rigid body. The discussion presupposes only an understanding of the elements of vector arithmetic and nowhere involves explicitly such higher concepts as tensor, screw, quaternion, etc. The treatment rests on a vector form of Rodrigues' formula which connects the initial and terminal positions of a point of a rigid body when the body is subjected to a displacement leaving one point fixed. This is first employed to furnish a simple vector proof of Euler's theorem on the displacements of a rigid body with one fixed point. Rodrigues' formula next yields a very simple demonstration of the formula for composition of finite rotations of a body with one fixed point. Chasles' theorem that the most general displacement of a rigid body may be effected by a translation along an axis followed by a rotation about that axis is next proved, followed by a derivation of the formula for the composition of such twists. All these are then extended to the corresponding theorems on infinitesimal displacements. (Received August 1, 1931.)

316. Professor E. V. Huntington: A simplified theory of the figure of the earth. (Preliminary communication.)

This paper shows that a rigorous formula for the figure of the earth (the theoretical form which the rotating earth would have if its surface were smooth) can be developed without any serious assumptions in regard to the distribution of matter inside the earth, and without using any mathematical theory more advanced than the calculus. (The theory of potential, and Clairaut's theorem are not required.) By using the concept of the (variable) center of attraction of the earth corresponding to any point on the surface, the parametric equations of the generating curve of the earth-revolute are found to be $x=a \cos \theta$, $y=b \sin \theta\left(1+f k \cos ^{2} \theta\right)$, where $a=$ the equatorial radius, $b=$ the polar radius, 
$f=(a-b) / a$, and $k=$ a new constant, which may be called the redundance of the earth, given by $f k / 4=\left(r_{q}-R_{q}\right) / a$, where $r_{q}$ is the quadrantal radius vector of the earth-curve, and $R_{q}$ is the quadrantal radius vector of an ellipse having the same semi-axes. If the earth were an ellipsoid of revolution, $k$ would be zero. The paper gives the necessary formulas for determining the constants $a, b$, and $r_{q}$ (or $a, f$, and $k$ ) in terms of measurements made on the surface of the earth, and for connecting the parameter $\theta$ with the observable latitude $\phi$; and also a formula for " $g$ " as a function of the latitude. (Received August 18, 1931.)

317. Professor E. H. Hildebrandt: Systems of polynomials connected with the Pearson differential and difference equations.

This paper is based on the fact that if $y$ is a non-identically vanishing solution of the Pearson differential equation $\left(b_{0}+b_{1} x+b_{2} x^{2}\right) d y / d x=\left(a_{0}+a_{1} x\right) y$, then the expression $\left(b_{0}+b_{1} x+b_{2} x^{2}\right)^{n} d^{n} y / d x^{n}$ when divided by $y$ defines a polynomial of degree at most $n$, and consequently a system of polynomials. The Hermite, Laguerre and Jacobi polynomials are special cases of such systems. On the basis of their definition, in terms of the differential equation above and the derivative process, a set of relations, differential and recurrence equations, can be deduced including as instances the well known relations for the special classes of polynomials mentioned. The second part of the paper discusses a similar process with respect to the difference equation $\left(b_{0}+b_{1} x+b_{2} x^{2}\right) \Delta u$ $=\left(a_{0}+a_{1} x\right) u$ and deduces relations parallel to those of the first part of the paper. (Received August 1, 1931.)

318. Professor P. R. Rider: On the distribution of the correlation coefficient in small samples.

This paper studies the frequency distribution of values of the correlation coefficient computed from small random samples from various bivariate populations. All evidence seems to point to the conclusion that the type of the sampled population has very little to do with the distribution, which is essentially the same as if the samples were drawn from a normal population. (Received August 7,1931.)

\section{Professor A. H. Copeland: The probability limit theorem.}

This paper is based on the concept of the Kollektiv introduced by von Mises. Each Kollektiv defines a probability distribution function. Corresponding to the operation of averaging a group of measurements, there is a law for combining the distribution functions so as to obtain the distribution function for the average. There are many elegant proofs showing that the resulting distribution function approaches the normal law as the number of measurements becomes infinite. We are not, however, justified in asserting that the resulting: Kollektiv defines this distribution function. As it is the Kollektiv which is of physical importance, an essential part of the reasoning has been omitted. This paper contains a discussion of the conditions under which the above assertion is justified. There is also a simple derivation of the probability limit theorem requiring no assumption of the existence of moments higher than the second. (Received August 10, 1931.) 
320. Dr. G. L. Edgett: Frequency distributions with given statistics which are not all moments.

A frequency curve has many well known statistics. The term statistics is here used for what is sometimes called a statistical constant or statistical parameter. Some of these may be expressed in terms of moments and some may not. In fitting analytic curves to observed distributions usually a curve is found for which the moments of the observed data are the same as the moments of the analytic curve. In this paper analytic curves are found having statistics in common with the observed data which are not all moments or statistics reducible to moments. Three different methods of finding such analytic curves are considered: first, by means of a differential equation; second, by means of a transformation of the normal curve of error; and, third, by means of a converging series. By the first method five analytic curves were found having given statistics in common with the raw data; by the second method, four analytic curves were found; by the third method, two analytic curves were found. The statistics used were some combination of the mean, standard deviation, mode, median, first quartile, and third quartile. (Received August 8, 1931.)

\section{Dr. C. C. Craig (National Research Fellow): On the Tchebycheff inequality of Bernstein.}

Tchebycheff's inequality in mathematical statistics gives for the probability that the deviation $x$ of a quantity from its mean shall not exceed in absolute value a given multiple of its standard deviation a lower limit which is often unsatisfactory. Among others, S. Bernstein has imposed conditions on the distribution of $x$ which lead to an improved limit, but his work, being published in Russian, has escaped attention outside of Russia. He showed that if $x$ is the sum of the independent variables, $x_{1}, x_{2}, \cdots, x_{n}$, and if the mathematical expectation of $\left|x_{i}\right|^{k}$ is $\leqq\left(\sigma_{x i}^{2} / z\right) k ! h^{k-z}$ for $k \geqq z, i=1,2, \cdots, n$, in which $\sigma_{x_{i}}$ is the standard deviation of $x_{i}$ and $h$ is arbitrary, then the probability $P$ of $|x|<t \sigma$ satisfies $P \geqq 1-z e^{\mu}$, where $\mu=-t^{2} /(2+2 h t / \sigma)$. The present paper briefly reproduces Bernstein's development and then shows that the same inequality can be reached under considerably less severe conditions; namely, that either the moments or the semi-invariants of $x$ obey the above condition on the absolute moments and that the tails of the frequency law for $x$ are wellbehaved. (Received August 10, 1931.)

322. Professor Raymond Garver: Two notes on the mathematics of finance.

This paper consists of two unrelated parts. The first considers the application of addition-subtraction logarithms to a number of computations, particularly that of interest rates in annuity problems. The second gives a formula for evaluating a certain type of property which is a generalization of the well known formula for the evaluation of wasting assets. (Received August 10, 1931.)

323. Professor E. T. Bell: Arithmetical composition and inversion of functions over classes. 
The compositions referred to depend upon two operations satisfying the associative law. The range of the independent variables is general, or abstract. This introduces a new type of number which is a matrix of any number of rows and columns, the elements in a given row being compounded according to a specific composition of the alove kind, and the compositions for the several rows being not necessarily identical. Such numbers include rational integers as a very special case. When the number of decompositions of a fixed element is finite, the composition is said to be arithmetical. This corresponds to unique factorization in arithmetic. Given a function whose arguments are abstract, except that decomposition is arithmetical as above, it is required to decompose the function into a composite of similar functions. This is the problem of inversion. A complete solution is given, which includes all of the numerous arithmetical inversions in the literature, and which provides the means for devising an infinity of new inversion processes. The paper will appear in the Transactions of this Society. (Received August 13, 1931.)

\section{Professor Tibor Rado: On the uniqueness of the solution of the problem of Plateau.}

The object of this paper is to generalize a uniqueness theorem given previously by the author (Proceedings of the National Academy of Sciences, vol. 16 (1930), p. 247), and to point out new examples of cases where the solution is not unique. (Received August 21, 1931.)

\section{Professor G.T. Whyburn: Concerning S-regions in locally connected continua.}

Let $M$ denote a locally connected compact metric continuum and call any region (= connected open set) in $M$ which has property $S$ (that is, which is the sum of a finite number of arbitrarily small connected sets) an $S$-region. The following sequence of theorems is established. (1) If $p$ is any point of $M$ and $R_{\epsilon}$ is the set of all points $x$ of $M$ which can be joined to $p$ by a simple chain of connected sets $L_{1}, L_{2}, \cdots, L_{n}$, where $\delta\left(L_{i}\right)<\epsilon-\sum_{1}^{\prime} \delta\left(L_{j}\right)$, then $R_{\epsilon}$ is an $S$-region; furthermore if $\sigma<\epsilon, \bar{R}_{\sigma} \subset R_{\epsilon}$. (2) Each point of $M$ is contained in an arbitrarily small $S$-region whose exterior has only a finite number of components. (3) Any region in $M$ with a totally disconnected boundary is an $S$-region. (4) Every region in $M$ is homeomorphic with an $S$-region in some $M$ space. (5) If $M$ has no local separating point, then every point $x$ of $M$ is contained in an arbitrarily small $S$-region $R$ such that $\bar{R}$ has no local separating point. (Received August 25, 1931.)

\section{Dr. Eberhard Hopf: Theory of measure and invariant} integrals.

This paper is concerned with the intrinsic characterization of transformations which possess an invariant integral. Let $M$ be a manifold and let $T$ be a 1-1 transformation of $M$ into itself, which carries measurable point sets and sets of zero measure into point sets of the same kind, respectively. An invariant integral may be associated with such a transformation $\int_{a} f(P) d m=m^{*}(a)$, $m^{*}(a)=m^{*}(T(a))$, where $f(P)$ is measurable and positive almost everywhere, 
while $a$ is any measurable set of points. Transformations of this kind are well known to play an important rôle in dynamics. Two measurable point sets $a$ and $a^{\prime}$ are called images by division of each other if $a$, as well as $a^{\prime}$, can be subdivided into denumerably many parts, so that each part of $a^{\prime}$ is an image of a part of $a$ under $T$ or a proper power of $T$, and conversely. The paper contains a proof of the following theorem. A necessary and sufficient condition for the existence of a finite invariant integral over $M$ is that $m\left(M-M^{\prime}\right)=0$, for each image by division $M^{\prime}$ of $M$. (Received August 27,1931.)

\section{Professor G. C. Evans: Poisson's equation with relation} to sub-harmonic functions.

The author continues the study of Poisson's equation in the form of Gauss's law for an arbitrary distribution of finite mass in a region $T$ bounded by a simple closed curve $C$, the latter having a continuously turning tangent such that the angle between neighboring tangents is dominated by a constant times the element of arc. He shows that $U_{1}=\int_{T} \log \frac{1}{2} d \phi(e)$, where $\phi(e)$ is the mass function, takes on summable boundary values for approach along normals to the boundary $C$ at almost all points, and that the absolute continuity of $\int U_{1} d s$ along arcs of curves in $T$, near $C$, is uniform over a sequence of curves which tends to $C$. Thus he is able to specify, as necessary and sufficient conditions, classes of functions in which the ordinary and generalized discontinuous Dirichlet problems are uniquely solvable. The generalized Newmann problem, in which the total flux is given on arcs of the boundary, is also uniquely solvable; but the problem is apparently too general to allow a determination in terms of the normal derivatives themselves. The methods apply to an arbitrary number of dimensions, and depend essentially on the fact that $U_{1}$ is the difference of two sub-harmonic functions.

\section{Dr. Selby Robinson: Covering theorems in general topology.}

Generalizations of the Borel theorem to abstract spaces are usually in the form of necessary and sufficient conditions for the reducibility of a proper covering of a set E. Such conditions involve either nuclearity or the closure of decreasing sequences of sets. In the present paper a study is made in general topological spaces of the reducibility of a covering to another of the same or a different kind, and of the reducibility of a covering of the space to a covering of a given set. Thus a number of new equivalences are found. The results are applied in connection with the generalization and extension of certain equivalences due to Sierpinski. A necessary and sufficient condition for the absolute closure of a space is extended to general topological spaces.

\section{Professor A. A. Albert: Normal division algebras of order sixteen over an algebraic field.}

The most important algebras for their applications are normal division algebras over algebraic fields $R(x)$ where $x$ is a root of an equation with rotational coefficients and irreducible in $R$, the field of all rational numbers. All normal division algebras of order four and nine have been shown by L. E. 
Dickson and J. H. M. Wedderburn respectively to be cyclic (Dickson) algebras. In the present paper the author completes the theory of the next case by showing that all normal division algebras of order sixteen over $R(x)$ are cyclic (Dickson) algebras. (Received July 27, 1931.)

\section{Professor J. A. Shohat: On interpolation.}

In the Göttinger Nachrichten for 1916 (Ueber Interpolation, pp. 66-91) Fejér has given an interpolation formula (different from Lagrange's) which possesses many interesting convergence properties, if the points used therein are zeros of Legendre or trigonometric polynomials, whose degree increases indefinitely. The present paper shows that the aforesaid convergence properties hold if the points used are zeros of any of the classical orthogonal polynomials (of Jacobi, Laguerre, Hermite). The analysis, based upon the theory of mechanical quadratures, yields some interesting convergence properties also for the ordinary Lagrange interpolation formula. (Received September 9, 1931.)

331. Mr. Alexander Tartler: On a certain new class of orthogonal polynomials.

It is known that in the theory of orthogonal Tchebycheff polynomials, defined by $\int_{a}^{b} \phi_{m}(x) \phi_{n}(x) d \psi(x)=\delta_{m n}$, the characteristic function $\psi(x)$ is assumed to be non-decreasing in the interval considered. Recently, Professor J. A. Shohat announced (Comptes Rendus, vol. 191, p. 474) without proof certain theorems and formulas for the case when $\psi(x)$ is no longer monotonic. The purpose of the present paper is to demonstrate his results and to carry the investigation further, particularly when $d \psi_{1}(x) \equiv(x-\alpha) d \psi(x), a<\alpha<b$. Necessary and sufficient conditions are given for the existence of a uniquely determined system of orthogonal and normal polynomials $\left\{\phi_{n}(x)\right\}, n=0,1$, $2, \cdots$. The distribution of the zeros of $\left\{\phi_{n}(x)\right\}$ and their dependence on $\alpha$ is investigated. Application to mechanical quadratures and certain asymptotic properties are given. Finally the case when $d \psi_{1}(x) \equiv(x-\alpha)(x-\beta) d \psi(x), a<\alpha$, $\beta<b$, is considered. (Received September 9, 1931.)

\section{Professor H. S. White: Seven lines in the real projective plane.}

When straight lines are continuous at infinity, seven lines with no three on a point divide the real plane into twenty-two convex and void polygons. If none of these are hexagons or heptagons, these are of only three types or species, all actual; passage from one type to another, under the group of all rotations of the seven lines, being possible only through a coincidence of two lines or of three points. Proof is deductive for four, five, and six lines, partly observational for a seventh. The object is partly to initiate a study of Aronhold sets of bitangents to a quartic. (Received September 9, 1931.)

\section{Mr. C. Winston: On mechanical quadratures of Gauss'} type. 
This paper consists of three parts. The first deals with a study of the coefficients $H_{i}$ in the mechanical quadrature formula

$$
\int_{a}^{b} f(x) p(x) d x=\sum_{i=1}^{n} H_{i} f\left(x_{i}\right), H_{i}=\int_{a}^{b}\left\{\phi_{n}(x) p(x) /\left[\left(x-x_{i}\right) \phi_{n}{ }^{\prime}\left(x_{i}\right)\right]\right\} d x,
$$

$\phi_{n}\left(x_{i}\right)=0$, where the $\phi_{n}(x)$ are orthogonal Tchebycheff polynomials corresponding to the characteristic function $p(x)$ and the interval $(a, b)$. Using a method due to Sonine (Memoirs, Russian Academy of Sciences, vol. 69(1892)) limits and order of magnitude, with respect to $n$, have been obtained for the $H_{i}$ for the classical orthogonal polynomials (of Legendre, Jacobi, Laguerre, and Hermite). The second part considers a mechanical quadratures formula of the above type with an arbitrary number of points $x_{i}$ fixed $\left(x_{i} \leqq a\right.$ or $\left.\geqq b\right)$. In the last part are found limits for the roots of the generalized Laguerre polynomials $\left(p(x)=e^{-x} x^{\alpha-1}, \alpha>0,(a, b)=(0, \infty)\right)$, following a method employed by $\mathrm{E}$. R. Neumann for the special case $\alpha=1$ (Jahresberichte der Deutschen Mathematiker-Vereinigung, vol. 30 (1921)). (Received September 9, 1931.) 